\title{
Values-based interprofessional education: how interprofessional education and values- based practice interrelate and are vehicles for the benefit of patients and health and social care professionals.
}

MERRIMAN, C., CHALMERS, L., EWENS, A., FULFORD, B. (KWF)., GRAY, R., HANDA, A. and WESTCOTT, L. 


\title{
Full version of short report
}

\section{Title}

Values-based Interprofessional Education: how Interprofessional Education and Values- based Practice interrelate and are vehicles for the benefit of patients and health and social care professionals.

\section{Authors:}

Clair Merriman, Oxford Brookes University, Oxford, UK OX8 OFP.

Laura Chalmers, Robert Gordon University, Aberdeen, UK.

Ann Ewens, Staffordshire University, Stafford, UK.

Bill (KWM) Fulford, Fellow and Director of the Collaborating Centre for Values-based Practice in Health and Social Care, St Catherine's College, Oxford, UK.

Richard Gray (corresponding author), richard.gray@caipe.org The Centre for the Advancement of Interprofessional Education, CAIPE, PO Box 680, Fareham, UK PO14 9NH.

Ashok Handa, Co-Director of the Collaborating Centre for Values-based Practice in Health and Social Care, Vascular Surgeon and Tutor, Nuffield Department of Surgical Sciences, St Catherine's College, Oxford UK.

Liz Westcott, Oxford Brookes University, Oxford, UK

\begin{abstract}
The 2016 All Together Better Health VIII Oxford conference brought together interprofessional education (IPE) and values-based practice (VBP) communities. As there is a paucity of research and publications in the area, following the event a working party consisting of representatives from both communities continued to meet and has developed a joint community of practice. This report describes the work achieved by the group so far and is intended for those involved in the planning and implementation of IPE and collaborative working. The authors consider that incorporating principles of VBP within a framework of IPE can provide a different perspective and understanding of the complexities involved in delivering realistic, student centred learning for collaborative practice, relevant in the 21st century workplace. In particular the authors suggest that using the principles of values and VBP in this way can inform the transition between IPE and collaborative practice facilitating effective person centered collaborative care. This process will require not only the incorporation of these principles within IPE sessions, but also incorporation within the training and support of new and established teachers involved in IPE.
\end{abstract}




\section{Key words:}

Interprofesssional Education; values-based practice; health and social care; collaborative

\section{Introduction}

There has been an IPE movement for several decades with The Centre for the Advancement of Interprofessional Education (CAIPE) being one of the national leaders in this area. However IPE has not gained the traction that would have been expected. To enhance the national standing of IPE, CAIPE led and was successful in its bid to host the international All Together Better Health conference in Oxford in 2016. CAIPE worked with Oxford Brookes University and the Collaborating Centre for Values-based Practice to develop a unique focus for the conference and bring together VBP and IPE. The planning and implementation of the conference successfully brought together the IPE and VBP communities. Representatives from 35 countries attended and the coproduction of ideas generated by the conference focus of IPE and VBP was positively received. Following the conference a new community of practice has emerged in this area. Given the policies such as the NHS long term plan which is looking at the next 10 years (NHS 2019), this emerging illumination of practice is important, inter alia, in supporting the development of the service models and skills required to deliver current standards of best practice in shared decision-making between professionals and patients in clinical care.

There is a paucity of both research and publications in this field and this short report will discuss our scholarly journey so far. It will reflect early thinking in this area on how IPE and VBP interrelate and are vehicles for the benefit of health and social care professionals, patients, service users, carers, families and those working with statutory, voluntary and independent organizations for care delivery.

\section{Values-based Practice}

VBP, like evidence-based practice (EBP), is a tool to support decision making. EBP provides a process that supports clinical decision-making where complex and conflicting evidence is in play. VBP provides a process that supports clinical decision-making where complex and conflicting values are in play. The two together support the model of shared clinical decision-making underpinning contemporary standards of consent to treatment (Fulford, Peile and Carroll, 2012) ${ }^{1}$.

VBP and EBP differ in the processes on which they rely. EBP relies on meta-analyses and other technical process elements. VBP relies on a combination of learnable clinical skills (covering awareness of values, reasoning, knowledge and certain aspects of communication skills) together with a particular service model (in which multidisciplinary teamwork provides the basis for person-centred care).

\footnotetext{
${ }^{1}$ For more information on values-based practice including a detailed reading guide and down-loadable resources go to valuesbasedpractice.org and access the link 'More about VBP.'
} 
The process of VBP also includes three principles marking particular aspects of its relationship with evidence based practice: the Two Feet Principle (that all clinical decisions including decisions about diagnosis depend on both values and evidence); the Squeaky Wheel Principle (that values and evidence become more noticeable when complex and conflicting but are always both in play); and the Science Driven Principle (that the need for VBP as well as EBP is driven by and increases with advances in medical science and technology). The process of VBP culminates in outputs that take the form of balanced decisions within frameworks of shared values.

VBP is sometimes thought to be important only where the evidence-base is lacking. But when it comes to clinical decision-making we really do need both. We need EBP to draw effectively on the science on which clinical interventions are based. We need VBP to link the science with the diverse values of (including anything that matters or is important to) individual patients and families (Fulford, Peile and Carroll, 2012).

\section{Interprofessional Education/Collaborative Practice}

The definition of IPE is well established by CAIPE. It is defined as 'occasions when two or more professions learn with, from and about each other to improve collaboration and the quality of care' (www.caipe.org). We see IPE as the vehicle for both students and practitioners to rehearse and refine their understanding and effectiveness of collaborative practice in a safe environment. This can be through for example simulation of team working using authentic scenarios.

Collaborative practice is concerned with issues of shared decision making and involves a shared accountability, within a professional team, for making the best decision(s) (Fulford, Peile and Carroll, 2012).

The concept of VBP can be the glue that holds the processes of IPE and collaborative practice together. It is a vehicle to ensure that balanced shared decision making can be made considering the different perspectives and values of all those involved.

IPE using a VBP approach allows a rehearsal of shared balanced decision making to take place in a learning environment which can then be translated into effective collaborative practice.

\section{Relationship between Interprofessional Education and Practice and Values-based Practice}

It is important initially to explore further the interactive relationship between IPE and VBP.

"I have taken to the idea of IPE being a vehicle for VBP- a real light bulb moment for me" (quote from a participant attending a recent IPE/VBP workshop). 
There can be nothing more satisfying as an educator than when a moment of learning is achieved .The point of Kairos as described by Neighbour (1996) is a critical moment when something important is poised to happen and cognitive dissonance emerges with an accompanying awareness of resonance, solidarity and empathy. VBP with its constructive focus on dissensus (Fulford, Peile and Carroll, 2012) can be associated with this process, facilitating an insightful response and encouraging deep learning.

An illustration of how the principles of VBP can inform IPE is the "delusion of sameness". It is important when learning and working interprofessionally that participants acknowledge, understand and resonate with each other's values. It is often assumed that those from different professional backgrounds working in teams have similar values. However hidden values may also be present that only emerge when the team is challenged resulting in unanticipated disagreements blocking effective team communication. If time is made for these hidden values to be made explicit and acknowledged, (facilitating a point of Kairos), then the negative process can be broken with different values utilized as a positive resource for responding to the different values of individual patients (Fulford, Peile and Carroll, 2012).

An example of this process applied to end of life care is described by Fulford, Peile and Carroll (2012) and can be summarized as follows: JM was admitted to his local hospital and following emergency surgery was found to have a cancer of the colon with multiple secondaries and a poor prognosis of a few weeks. The surgeon considered the operation technically successful, and as he was keen for his patient to be moved, referred him to the oncologist for chemotherapy treatment. However as from an evidence based perspective there was no agreed consensus about the advantage of further treatment, the oncologist requested an opinion from the palliative care consultant. Meanwhile as there was difficulty with pain control and lack of home facilities, the nursing team and occupational therapist were concerned about sending JM home. The palliative care consultant spoke to the patient's wife who accepted that her husband's condition was incurable but was frightened about him coming home. He then spoke to JM who knew he was terminal and wished to die at home but had not spoken to his wife as he wished to protect her. The professionals caring for JM had worked well together for some years and had developed mutual respect but they all now had differing perspectives about the way forward. It was decided to hold a multi disciplinary team meeting for all professional involved. During the meeting, the palliative care consultant reflected the views of the patient and his wife. The views of all concerned were listened to, acknowledged and respected and allowed to remain fully in play. Through dissensus a balance of perspectives was achieved with values remaining central. Communication was encouraged between JM and his wife and he was discharged home with intensive home support. Control of pain was not a problem and JM decided to go for a lower dose palliative treatment regime.

Some would argue that VBP principles are already used within IPE but these are not always made explicit or articulated. Some participants may be unconsciously competent; others are not using it to an optimum level and some not at all. It is suggested that making the skills and principles of VBP explicit and using them in this way can bring a depth to collaborative decisions, reducing the effects of hierarchy, promoting equality whilst acknowledging personal, professional and interprofessional values resulting in more balanced decision making and improved outcomes. 
The time is right to acknowledge that IPE is not and never should be a separate entity, (Barr et al 2006) discipline or subject. It is suggested that by using VBP within the structure of IPE can result in effective collaborative working and by means of this, produce a greater emphasis on person centred, collaborative care.

\section{Background to the Values-based Practice Group}

The Oxford 2016 All Together Better Health Conference VIII conference was entitled "Values-based Interprofessional Education and Practice". The conference steering group agreed after the event to continue developments by implementing a series of annual workshops with the purpose of clarifying the relationship between IPE and VBP. One of the outcomes has been the implementation of a Values-based Interprofessional Education and Practice Network as part of the Collaborating Centre. Aims of the network include extending the community of practice and providing mutual support and practical help for development of relevant initiatives. The network welcomes new members and further information can be foundathttps://valuesbasedpractice.org/what-do-we-do/networks/If you wish to join please email contact@valuesbasedpractice.org

\section{Conclusion}

This short report discusses our thinking about the relationship between IPE and VBP. It is already acknowledged that undertaking IPE allows participants to develop their understanding and the effectiveness of their collaborative working. This report makes an additional contribution by suggesting that the incorporation of the principles and process of VBP, within the structure of IPE, can constructively inform this relationship with collaborative practice. In essence it facilitates the movement from IPE to more person centred collaborative care. This has important implications not only for the use of the principles of VBP within IPE but also for inclusion within the preparation and support curriculum of both new and established teachers involved in IPE.

\section{References}

Barr, H., Freeth, D., Hammick, M., Kopel, I., Reeves, S. (2006). The evidence base and recommendations for interprofessional education in health and social care.Journal of Interprofessional Care. 2091 75-78.

Fulford, K.,Peile,E., and Carroll, H (2012) Essential Values-Based Practice. Page 42. Cambridge University Press, Cambridge.

Neighbour, R (1996) The Inner Apprentice. Pages 135-137.Petroc Press, Newbury.

National Health Service (2019) The NHS Long Term Plan https://www.longtermplan.nhs.uk/wpcontent/uploads/2019/01/nhs-long-term-plan.pdf 